\title{
Energy Reduction with Anticontrol of Chaos for Nonholonomic Mobile Robot System
}

\author{
Zahra Yaghoubi, ${ }^{1}$ Hassan Zarabadipour, $^{1}$ \\ and Mahdi Aliyari Shoorehdeli ${ }^{2}$ \\ ${ }^{1}$ Department of Electrical Engineering, Imam Khomeini International University, \\ Qazvin 34149-16818, Iran \\ ${ }^{2}$ Department of Mechatronics Engineering, Faculty of Electrical Engineering, \\ K. N. Toosi University of Technology, Tehran 19697, Iran \\ Correspondence should be addressed to Zahra Yaghoubi, mn_y3567@yahoo.com
}

Received 18 June 2012; Accepted 7 September 2012

Academic Editor: Jinhu Lü

Copyright (C) 2012 Zahra Yaghoubi et al. This is an open access article distributed under the Creative Commons Attribution License, which permits unrestricted use, distribution, and reproduction in any medium, provided the original work is properly cited.

\begin{abstract}
This paper presents energy reduction with anticontrol of chaos for nonholonomic mobile robot system. Anticontrol of chaos is also called chaotification, meaning to chaotify an originally nonchaotic system, and in this paper error of mobile robot system has been synchronized with chaotic gyroscope for reducing energy and increasing performance. The benefits of chaos synchronization with mechanical systems have led us to an innovation in this paper. The main purpose is that the control system in the presence of chaos work with lower control cost and control effort has been reduced. For comparison of proposed method, the feedback linearization controller has also been designed for mobile robot with noise. Finally, the efficacies of the proposed method have been illustrated by simulations, energy of control signals has been calculated, and effect of Alpha $(\alpha$ : a constant coefficient is used beside of chaotic system) variations on the energy of control signals has been checked.
\end{abstract}

\section{Introduction}

Wheeled mobile robots (WMRs) have been an active area of research and development over the past three decades [1]. This long-term interest has been mainly fueled by the myriad of practical applications that can be uniquely addressed by mobile robots due to their ability to work in large domains. These WMRs are called nonholonomic mobile robots because of their no-slip kinematic constraints. Nonholonomic behavior in robotic systems is particularly interesting because it implies that the mechanism can be controlled with reduced number of actuators [2]. 
The tracking control approaches for the mobile robots are mainly divided into six types: (1) feedback linearization [3-5]; (2) sliding-mode control [6, 7]; (3) backstepping control [8-11]; (4) computed torque; (5) adaptive control [12, 13]; (6) intelligent control [14].

Tracking control by using synchronization of a mechanical system with a chaotic system is presented in this paper and it is compared with error and with noise.

Many natural and man-made systems can be represented by means of a graph in mathematical terms, and these graphs are called complex networks. Most real-world industrial systems such as mobile robot are typical complex networks. Collective motions of complex networks have recently been the subject of considerable interest within the science and technology communities. Especially, one of the interesting and significant phenomena in complex dynamical networks is the synchronization of all dynamical nodes in a network $[15,16]$. There are many papers which discuss the network synchronization problem, and there are results reported in the literature on how to rigorously characterize the network synchronizability [17].

Dynamic chaos is a very interesting nonlinear effect which has been intensively studied during the last three decades. Chaos control can be mainly divided into two categories [18]: one is the suppression of the chaotic dynamical behavior and the other is to generate or enhance chaos in nonlinear system. Because of features of chaos system such as globally stable, we use it, such that the error system is synchronized with chaotic system and state variables follow the reference path. Error synchronization is also called anti-control of chaos or chaotification meaning to chaotify an originally non-chaotic system.

For common tracking control, error converges to zero, but, generally, there are three choices for error: error converges to zero, error is synchronized with periodic signals, and error is synchronized with chaotic system.

In this paper, error of mobile robot system is synchronized with chaotic gyroscope for reducing energy and increasing performance. For comparison of proposed method, the feedback linearization controller is also designed for mobile robot with Gaussian and Rayleigh noises.

The paper is organized in the following manner. Nonholonomic mobile robot dynamics and its state-space representation and the constraint equations of the mobile robot are described in Section 2. In Section 3, generally, the feedback linearization controller for MIMO (multi-input-multi-output) nonlinear systems is explained. Section 4 presents the feedback linearization controller for mobile robot. In Section 5, anti-control of chaos and feedback linearization controller for mobile robot by using chaotification are presented. In Section 6, chaotic gyroscope system is described. Feedback linearization for mobile robot with noise is presented in Section 7. In Section 8, simulation results for control of mobile robot, anti-control of chaos for control mobile robot, control of the mobile robot with noise, comparison between them, and effect of Alpha ( $\alpha$ : a constant coefficient is used beside of chaotic system) variations on the energy of control signals are presented, and the final section is conclusion.

\section{Mobile Robot Kinematics}

The kinematic model of the mobile robot is given as follows:

$$
\left[\begin{array}{l}
\dot{x} \\
\dot{y} \\
\dot{\theta}
\end{array}\right]=\left[\begin{array}{cc}
\cos \theta & 0 \\
\sin \theta & 0 \\
0 & 1
\end{array}\right] \cdot\left[\begin{array}{c}
\mathcal{v} \\
\omega
\end{array}\right],
$$




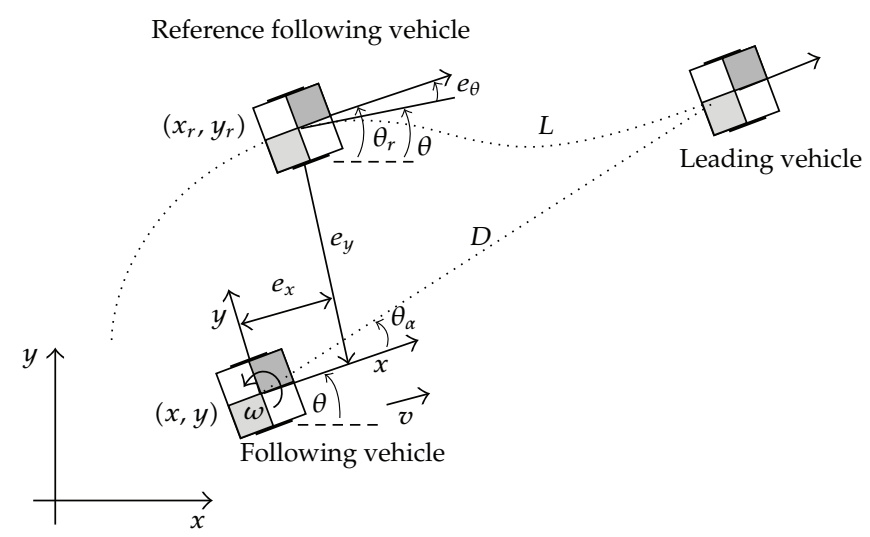

Figure 1: Illustration of the error transformation where the following vehicle follows the path of the leading vehicle at distance $L$.

where $v$ and $\omega$ are the forward and the angular velocities are considered as inputs, (following vehicle in Figure 1). The no-slip condition, and, namely, no lateral velocity, imposes the nonholonomic constraint:

$$
\dot{x} \sin \theta+\dot{y} \cos \theta=0 .
$$

Assume that a feasible reference $\left(x_{r}, y_{r}, v_{r}, \omega_{r}\right)^{T}$ is given and satisfies the following equations:

$$
\left[\begin{array}{c}
\dot{x}_{r} \\
\dot{y}_{r} \\
\dot{\theta}_{r}
\end{array}\right]=\left[\begin{array}{cc}
\cos \theta_{r} & 0 \\
\sin \theta_{r} & 0 \\
0 & 1
\end{array}\right] \cdot\left[\begin{array}{c}
v_{r} \\
\omega_{r}
\end{array}\right]
$$

where $v_{r}$ and $\omega_{r}$ are considered as reference inputs and they can be derived as

$$
v_{r}=\sqrt{\dot{x}_{r}^{2}+\dot{y}_{r}^{2}}, \quad \omega_{r}=\frac{\dot{x}_{r} \ddot{y}_{r}-\dot{y}_{r} \ddot{x}_{r}}{\dot{x}_{r}^{2}+\dot{y}_{r}^{2}}
$$

In Figure 1 the reference vehicle is an imaginary vehicle that ideally follows the reference path. In contrast, the real vehicle (when compared to the reference vehicle) has some error when following the reference path. The trajectory tracking error, expressed in terms of the following vehicle, as shown in Figure 1, is given by [19]

$$
e=\left[\begin{array}{l}
e_{x} \\
e_{y} \\
e_{\theta}
\end{array}\right]=\left[\begin{array}{ccc}
\cos \theta & \sin 0 & 0 \\
-\sin \theta & \cos 0 & 0 \\
0 & 0 & 1
\end{array}\right] \cdot\left[\begin{array}{l}
x_{r}-x \\
y_{r}-y \\
\theta_{r}-\theta
\end{array}\right]
$$


Differentiating (2.5) with respect to time and taking into account the kinematic model given by (2.1), and the equivalent kinematic model of the reference vehicle given by (2.3), the following nonlinear error model of the system is obtained:

$$
\left[\begin{array}{c}
\dot{e}_{x} \\
\dot{e}_{y} \\
\dot{e}_{\theta}
\end{array}\right]=\left[\begin{array}{c}
v_{r} \cos e_{\theta} \\
v_{r} \sin e_{\theta} \\
\omega_{r}
\end{array}\right]+\left[\begin{array}{cc}
-1 & e_{y} \\
0 & -e_{x} \\
0 & -1
\end{array}\right]\left[\begin{array}{c}
v \\
\omega
\end{array}\right]
$$

Here, the tracking control problem is to find appropriate control laws for $v$ and $\omega$ such that the tracking error $\left(e_{x}, e_{y}, e_{\theta}\right)^{T}$ converges to zero.

\section{Feedback Linearization for MIMO Nonlinear Systems}

Consider a "square" system (where the number of inputs is equal to the number of outputs $=m)$ :

$$
\begin{aligned}
\dot{x} & =f(x)+\sum_{i=1}^{m} g_{i} \cdot u_{i}, \\
y & =\left[h_{1}, \ldots, h_{m}\right]^{T}, \\
\dot{y}_{k} & =L_{f}\left(h_{k}\right)+\sum_{i=1}^{m} L_{g_{i}}\left(h_{k}\right) u_{i} .
\end{aligned}
$$

Let $r_{k}$, the relative degree, be defined as the relative degree of each output, that is, for some $i$, $L_{g_{i}}\left(L_{f}^{r_{k}-1}\left(h_{k}\right)\right) \neq 0$.

Let $J(x)$ be a $m \times m$ matrix such that:

$$
J(x)=\left[\begin{array}{ccc}
L_{g_{i}}\left(L_{f}^{r_{1}-1}\left(h_{1}\right)\right) & \cdots & L_{g_{m}}\left(L_{f}^{r_{1}-1}\left(h_{1}\right)\right) \\
\cdots & \cdots & \cdots \\
L_{g_{i}}\left(L_{f}^{r_{m}-1}\left(h_{m}\right)\right) & \cdots & L_{g_{m}}\left(L_{f}^{r_{m}-1}\left(h_{m}\right)\right)
\end{array}\right]
$$

$J(x)$ is called the decoupling matrix. We will assume that $J(x)$ is nonsingular.

Let

$$
y^{r}=\left[\begin{array}{c}
\frac{d^{r_{1}} y_{1}}{d t^{r_{1}}} \\
\vdots \\
\frac{d^{r_{m}} y_{m}}{d t^{r_{m}}}
\end{array}\right]
$$




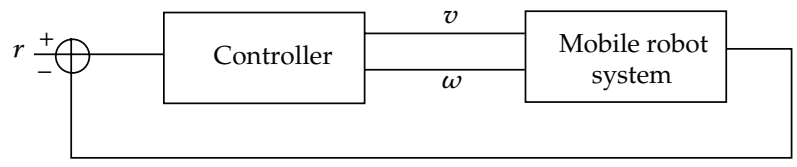

Figure 2: Block diagram of control system.

where $y^{r}$ is an $m \times 1$ vector:

$$
l(x)=\left[\begin{array}{c}
L_{f}^{r_{1}}\left(h_{1}\right) \\
\vdots \\
L_{f}^{r_{m}}\left(h_{m}\right)
\end{array}\right] .
$$

Then we have

$$
y^{r}=l(x)+J(x) \cdot u=v,
$$

where $v$ is the synthetic input ( $v$ is $m \times 1)$.

Now, we obtain a decoupled set of equations:

$$
\begin{gathered}
\frac{d^{r_{1}} y_{1}}{d t^{r_{1}}}=v_{1} \\
\quad \vdots \quad \text { so } y \Longleftrightarrow v, \\
\frac{d^{r_{m}} y_{m}}{d t^{r_{m}}}=v_{m} .
\end{gathered}
$$

To obtain the control $u$, design $v$ by using linear techniques:

$$
u=J^{-1}(v-l)
$$

\section{Feedback Linearization for Mobile Robot}

Considering the kinematic model of mobile robot given by (2.1), as shown in Figure 2, the feedback linearization controller will be designed.

$y_{1}=x_{1}$ and $y_{2}=x_{2}$ are defined as outputs:

$$
\left[\begin{array}{l}
\dot{y}_{1} \\
\dot{y}_{2}
\end{array}\right]=\left[\begin{array}{ll}
\cos \theta & 0 \\
\sin \theta & 0
\end{array}\right]\left[\begin{array}{l}
v \\
\omega
\end{array}\right]
$$

$J(x)=\left[\begin{array}{cc}\cos \theta & 0 \\ \sin \theta & 0\end{array}\right]$ is clearly singular (has rank 1$)$.

Let $v=x_{3}, \dot{v}=\dot{x}_{3}=u_{1}, \omega=u_{2}$. 


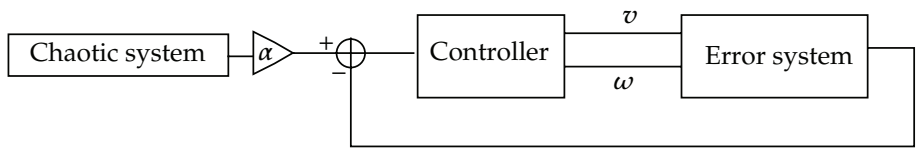

Figure 3: Block diagram of control system.

The state has been extended:

$$
\begin{aligned}
& \dot{x}_{1}=x_{3} \cos \theta, \\
& \dot{x}_{2}=x_{3} \sin \theta, \\
& \dot{x}_{3}=\dot{v}=u_{1}, \\
& \dot{\theta}=\omega=u_{2} .
\end{aligned}
$$

Take $y_{1}=x_{1}$ and $y_{2}=x_{2}$ :

$$
\left[\begin{array}{l}
\ddot{y}_{1} \\
\ddot{y}_{2}
\end{array}\right]=\left[\begin{array}{cc}
\cos \theta & -x_{3} \sin \theta \\
\sin \theta & x_{3} \cos \theta
\end{array}\right]\left[\begin{array}{l}
u_{1} \\
u_{2}
\end{array}\right] .
$$

The relative degrees are 2, and the new $J(x)$ matrix: $J(x)=\left[\begin{array}{cc}\cos \theta & -x_{3} \sin \theta \\ \sin \theta & x_{3} \cos \theta\end{array}\right]$ is nonsingular for $x_{3} \neq 0$ :

$$
\begin{aligned}
& \ddot{y}_{1}=V_{1}, \\
& \ddot{y}_{2}=V_{2} .
\end{aligned}
$$

To obtain the control, $u$ :

$$
\left[\begin{array}{l}
u_{1} \\
u_{2}
\end{array}\right]=J^{-1}(x)\left[\begin{array}{l}
V_{1} \\
V_{2}
\end{array}\right]=\left[\begin{array}{cc}
\cos \theta & \sin \theta \\
\frac{-1}{x_{3}} \sin \theta & \frac{1}{x_{3}} \cos \theta
\end{array}\right]\left[\begin{array}{l}
V_{1} \\
V_{2}
\end{array}\right],
$$

design $\left(V_{1}, V_{2}\right)^{T}$ by using linear techniques and $\dot{v}=u_{1}$.

\section{Feedback Linearization for Mobile Robot with Using Anticontrol of Chaos (Chaotification)}

In previous section, error signals are converged to zero but in this section the error signals track the small ratio of amplitude of chaotic gyroscope system, as shown in Figure 3. For making the small ratio of amplitude, a constant coefficient is used beside of chaotic system. This coefficient is $10^{-10} \leq \alpha \leq 1$. 
Feedback linearization technique in previous section will be used for error system is given by (2.6); $y_{1}=e_{y}$ and $y_{2}=e_{\theta}$ are defined as outputs:

$$
\left[\begin{array}{l}
\dot{y}_{1} \\
\dot{y}_{2}
\end{array}\right]=\left[\begin{array}{cc}
0 & e_{x} \\
0 & -1
\end{array}\right]\left[\begin{array}{c}
v \\
\omega
\end{array}\right]+\left[\begin{array}{c}
v_{r} \sin e_{\theta} \\
\omega_{r}
\end{array}\right]
$$

$J(x)=\left[\begin{array}{ll}0 & -e_{x} \\ 0 & -1\end{array}\right]$ is clearly singular (has rank 1$)$.
Let $=u_{1}, \omega=x_{4}, \dot{\omega}=\dot{x}_{4}=u_{2}$.

The state has been extended:

$$
\begin{gathered}
\dot{e}_{x}=v_{r} \cos e_{\theta}-u_{1}+e_{y} x_{4}, \\
\dot{e}_{y}=v_{r} \sin e_{\theta}+e_{x} x_{4}, \\
\dot{e}_{\theta}=\omega_{r}-x_{4}, \\
\dot{x}_{4}=\dot{\omega}=u_{2} .
\end{gathered}
$$

Take $y_{1}=e_{y}$ and $y_{2}=e_{\theta}$ :

$$
\left[\begin{array}{l}
\ddot{y}_{1} \\
\ddot{y}_{2}
\end{array}\right]=\left[\begin{array}{cc}
x_{4} & -e_{x} \\
0 & -1
\end{array}\right]\left[\begin{array}{l}
u_{1} \\
u_{2}
\end{array}\right]+\left[\begin{array}{c}
\left(-2 v_{r} x_{4}+v_{r} \omega_{r}\right) \cos e_{\theta}-e_{y} x_{4}^{2}+\dot{v}_{r} \sin e_{\theta} \\
\dot{\omega}_{r}
\end{array}\right]
$$

The relative degrees are 2, and the new $J(x)$ matrix: $J(x)=\left[\begin{array}{cc}x_{4} & -e_{x} \\ 0 & -1\end{array}\right]$ is nonsingular for $x_{4} \neq 0$, and

$$
\begin{aligned}
& l(x)=\left[\begin{array}{c}
\left(-2 v_{r} x_{4}+v_{r} \omega_{r}\right) \cos e_{\theta}-e_{y} x_{4}^{2}+\dot{v}_{r} \sin e_{\theta} \\
\dot{\omega}_{r}
\end{array}\right], \\
& \ddot{y}_{1}=V_{1} \text {, } \\
& \ddot{y}_{2}=V_{2} \text {. }
\end{aligned}
$$

To obtain the control, $u$ :

$$
\begin{aligned}
{\left[\begin{array}{l}
u_{1} \\
u_{2}
\end{array}\right] } & =J^{-1}(x)\left(\left[\begin{array}{l}
V_{1} \\
V_{2}
\end{array}\right]+l(x)\right) \\
& =\left[\begin{array}{c}
\frac{1}{x_{4}}\left(V_{1}+\left(2 v_{r} x_{4}-v_{r} \omega_{r}\right) \cos e_{\theta}+e_{y} x_{4}^{2}-\dot{v}_{r} \sin e_{\theta}-e_{x}\left(V_{2}-\dot{\omega}_{r}\right)\right) \\
-\left(V_{2}-\dot{\omega}_{r}\right)
\end{array}\right],
\end{aligned}
$$

design $\left(V_{1}, V_{2}\right)^{T}$ by using linear techniques and $\dot{\omega}=u_{2}$. 


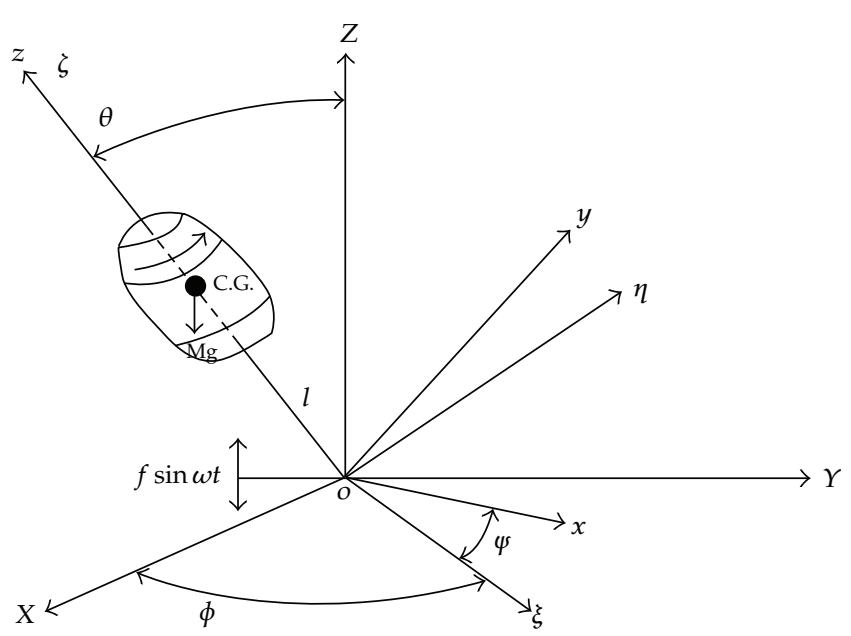

Figure 4: A schematic diagram of a symmetric gyroscope.

\section{Chaotic Gyroscope System}

The symmetric gyroscope mounted on a vibrating base is shown in Figure 4 . The dynamics of a symmetrical gyro with linear-plus-cubic damping of angle $\theta$ can be expressed as [20]

$$
\ddot{\theta}+\alpha_{1}^{2} \frac{(1-\cos \theta)^{2}}{\sin ^{3} \theta}-\beta_{1} \sin \theta+c_{1} \dot{\theta}+c_{2} \dot{\theta}^{3}=f \sin \omega t \sin \theta,
$$

where $f \sin \omega t$ is a parametric excitation, $c_{1} \dot{\theta}$ and $c_{2} \theta^{3}$ are linear and nonlinear damping terms, respectively, and $\alpha_{1}^{2}\left((1-\cos \theta)^{2} / \sin ^{3} \theta\right)-\beta_{1} \sin \theta$ is a nonlinear resilience force. According to [20], in a symmetric gyro mounted on a vibrating base, the precession and the spin angles have cyclic motions, and hence their momentum integrals are constant and equal to each other. So the governing equations of motion depend only on the mutational angle $\theta$. Using Routh's procedure and assuming a linear-plus-cubic form for dissipative force, (6.1) is obtained [20]. Given the states $x_{1}=\theta, x_{2}=\dot{\theta}$ and $g(\theta)=\alpha_{1}^{2}\left((1-\cos \theta)^{2} / \sin ^{3} \theta\right)-\beta_{1} \sin \theta$, (6.1) can be rewritten as follows:

$$
\begin{gathered}
\dot{x}_{1}=x_{2} \\
\dot{x}_{2}=g\left(x_{1}\right)-c_{1} x_{1}-c_{2} x_{2}{ }^{3}+\left(\beta_{1}+f \sin \omega t\right) \sin \left(x_{1}\right) .
\end{gathered}
$$

This gyro system exhibits complex dynamics and has been studied by [20] for values of $f$ in the range $32<f<36$ and constant values of $\alpha_{1}^{2}=100, \beta_{1}=1, c_{1}=0.5, c_{2}=0.05$, and $\omega=2$. Figure 5 illustrates the irregular motion exhibited by this system for $f=35.5$ and initial conditions of $\left(x_{1}, x_{2}\right)=(1,-1)$. 

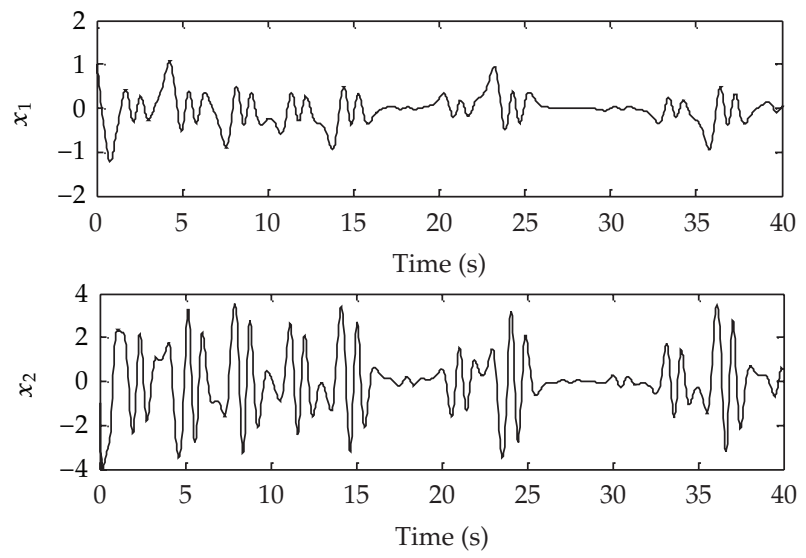

(a)

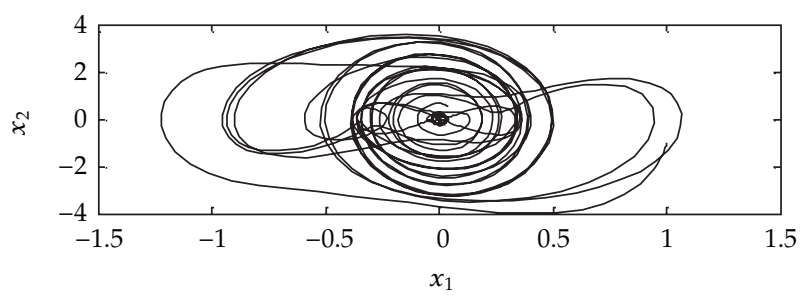

(b)

Figure 5: (a) Time series of $x_{1}$ and $x_{2}$, (b) phase portrait of gyroscope.

\section{Feedback Linearization for Mobile Robot with Noise}

As in previous section, instead of chaos system, noise is entered to control block diagram. It means that error system instead of converging to zero, is faced with noise, as shown in Figure 6.

System has two inputs and two outputs; in anti-control of chaos for desired references, the two states gyroscope system is used, and for noise, Gaussian noise and Rayleigh noise are the desired references for error system.

Equations are similar to Section 5, and for making the small ratio of amplitude, a constant coefficient is used beside of noise. This coefficient is $10^{-10} \leq a \leq 1$.

\section{Simulation Results}

\subsection{Control of the Mobile Robot}

Control of the mobile robot dynamic model is applied to system with circle feasible reference (desired), and results are shown in Figure 7. In this figure reference path is circle $\left(x_{r}=\cos t\right.$, $\left.y_{r}=\sin t\right)$. In Figure $7(a)$, control signals $(v$ and $\omega)$ for controlling mobile robot and, in Figure $7(b)$, path following by mobile robot are shown. 


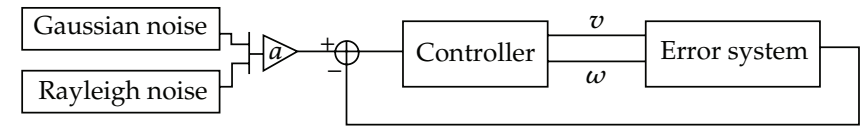

Figure 6: Block diagram of control system.
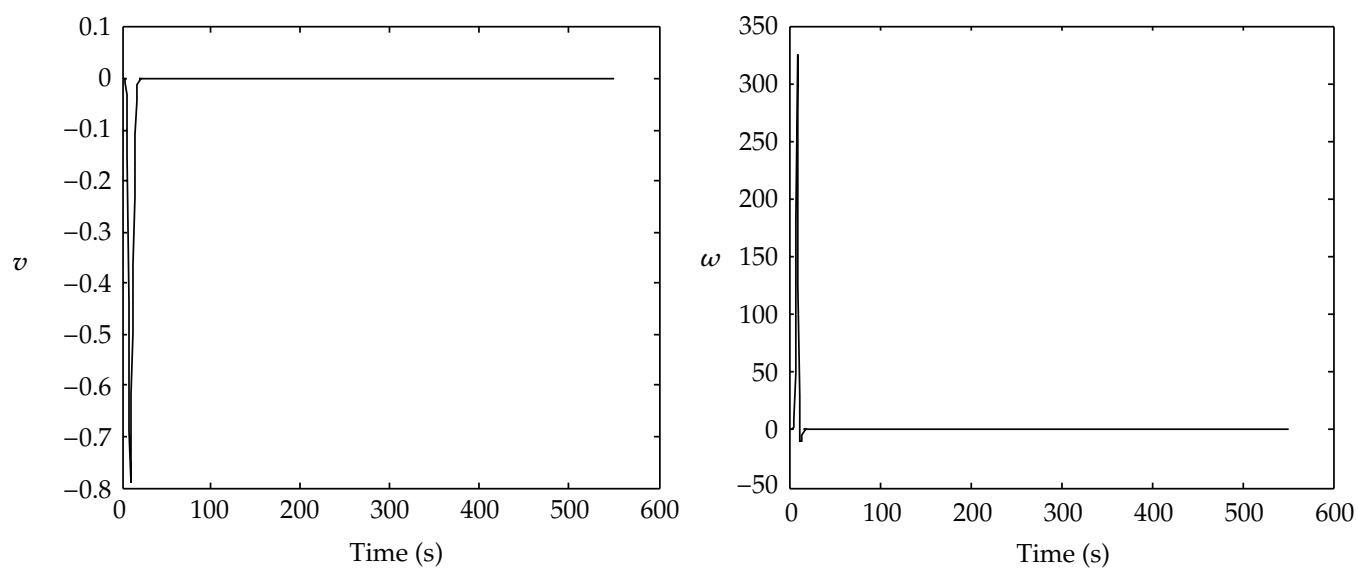

(a)

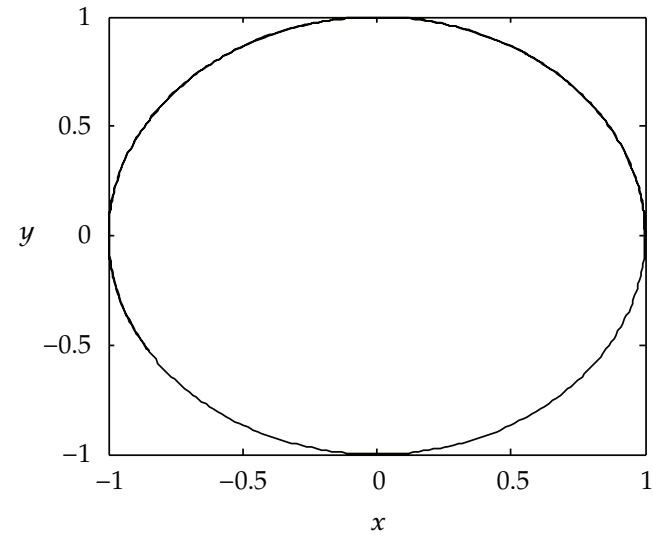

(b)

Figure 7: (a) Control signals for circle reference and (b) path following.

\subsection{Anticontrol of Chaos for Controlling Mobile Robot}

Anti-control of chaos for the mobile robot dynamic model is applied to system with circle feasible reference (desired), and results are shown in Figure 8. In this figure reference path is circle $\left(x_{r}=\cos t, y_{r}=\sin t\right)$. In Figure 8(a), control signals $(v$ and $\omega)$ for controlling mobile robot and, in Figure 8(b), path following by mobile robot are shown. As shown in Figure 3, the error system is synchronized with chaotic gyroscope systems and $\alpha=10^{-4}$. Gyroscope system has two states, and the error system has three states; for this problem, we synchronize second state of error system with second state of gyroscope system and third state of error system with first state of gyroscope system, and first state of error system commonly is tracked zero. 

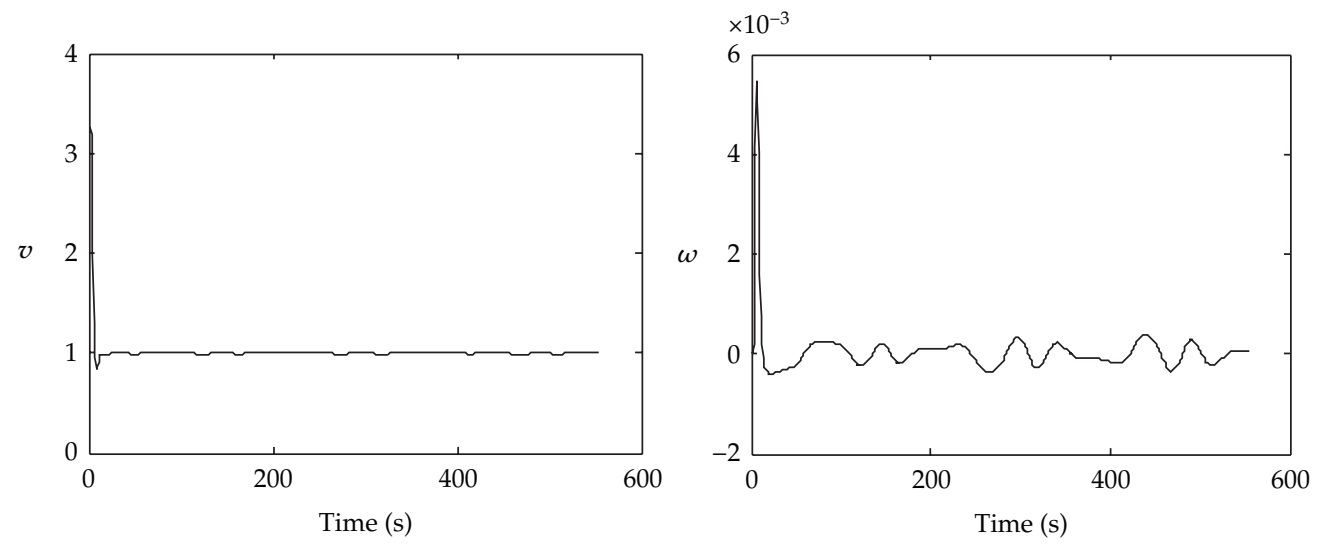

(a)

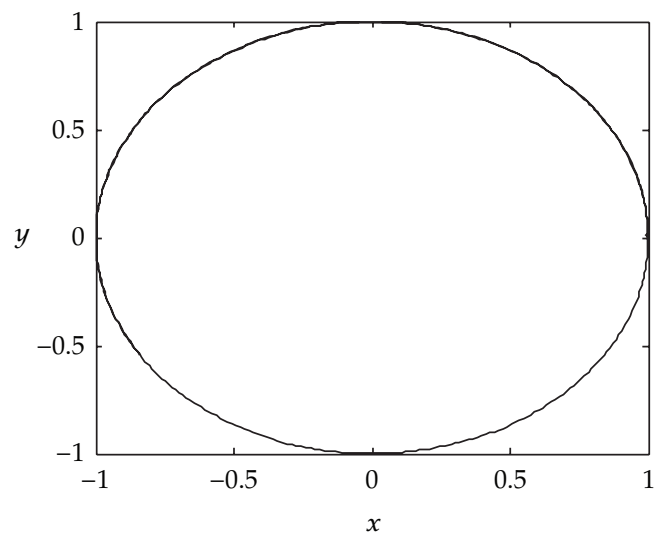

(b)

Figure 8: (a) Control signals for circle reference for anti-control of chaos with $\alpha=10^{-4}$ and (b) path following.

\subsection{Control of the Mobile Robot with Noise}

Control of the mobile robot with noise is applied to system with circle feasible reference (desired), and results are shown in Figure 9. In this figure reference path is circle $\left(x_{r}=\cos t\right.$, $\left.y_{r}=\sin t\right)$. In Figure 9(a), control signals $(v$ and $\omega)$ for controlling mobile robot and, in Figure $9(\mathrm{~b})$, path following by mobile robot are shown. As shown in Figure 6, the error system is converging to zero with Gaussian noise and Rayleigh noise and $a=10^{-4}$.

\subsection{Energy Comparison}

The major purpose of this paper is energy comparison between control signals. These control signals are for control of mobile robot and error system synchronization with chaos and control of error system with noise. In this paper, energy means square of signals integral (area under the curve); energy is calculated as follows:

$$
\text { energy }=\sum\left(v^{2}+\omega^{2}\right) \text {. }
$$



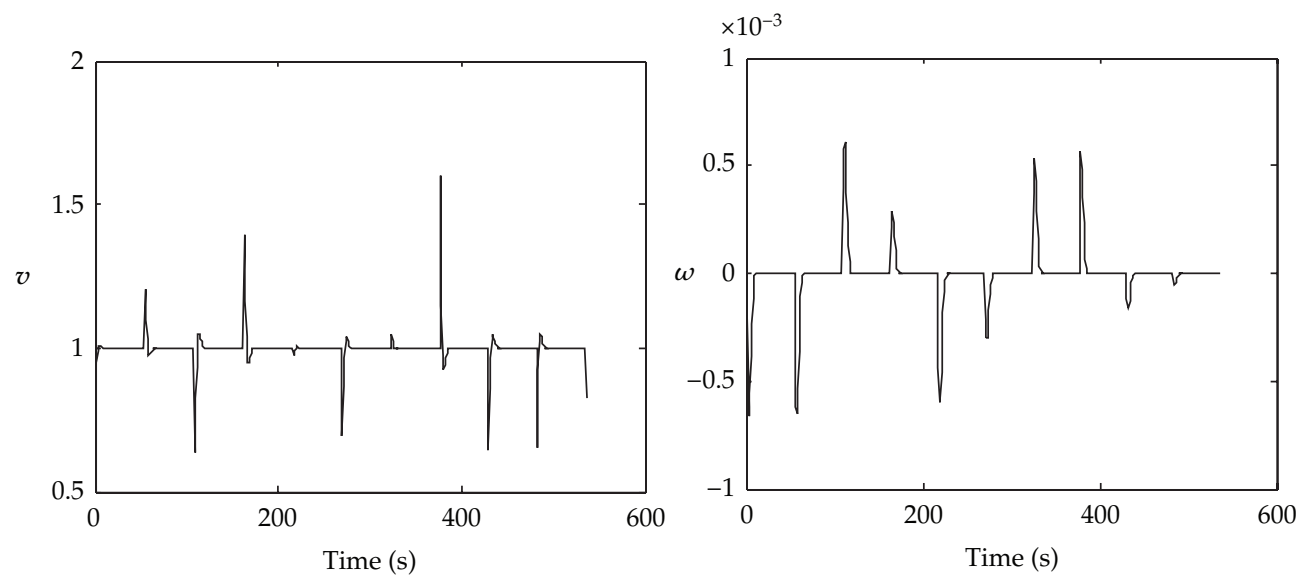

(a)

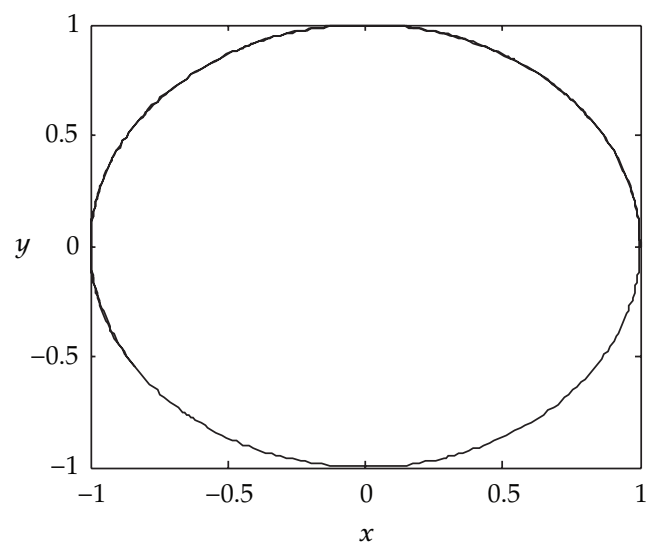

(b)

Figure 9: (a) Control signals for circle wave reference for noise with $a=10^{-4}$ and (b) path following.

Table 1: Energy comparison.

\begin{tabular}{lccc}
\hline Control & Feedback linearization control & Anti-control of chaos & Noise \\
\hline Circle & 15683 & 592.0880 & 674.5611 \\
\hline
\end{tabular}

The comparison of energy is shown in Table 1, it can be seen that anticontrol of chaos of error system synchronization with chaotic gyroscope and $\alpha=10^{-4}$ improves energy even better than error with noise.

\subsection{Effect of Alpha ( $\alpha$ ) Variations on the Energy of Control Signals}

In this section, we show the effect of Alpha $(\alpha)$ on energy of control signal with Figure 10. When Alpha $(\alpha)$ is zero (it means to control mobile robot that error converges to zero), energy value is more than energy values in $0<\alpha<10^{-3}$. For $\alpha<0$, energy increases from minimum value. 


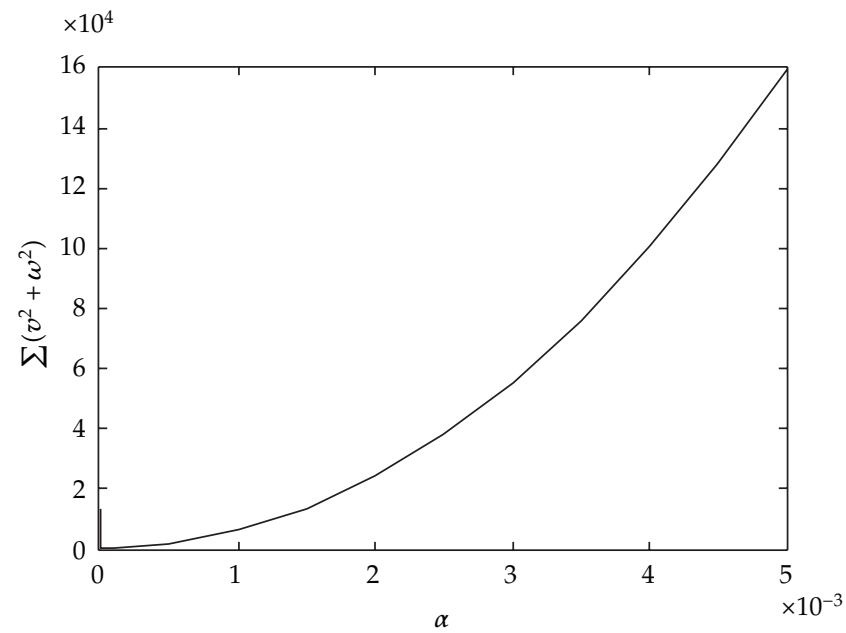

Figure 10: Effect of Alpha $(\alpha)$ variations on the energy of control signals.

\section{Conclusion}

In this paper, energy reduction with anti-control of chaos for nonholonomic mobile robot system was checked, and the mobile robot system was, controlled with three different ways and feedback linearization controller was used for controlling with circle feasible references. Error of mobile robot system was synchronized with chaotic system instead of zero tracking and was compared with noise instead of chaotic system. Energy improving for anti-control of chaos is visible. Finally, this mobile robot control with chaos synchronization also provides some new insights for controlling and the possible applications in the real-world engineering systems.

\section{References}

[1] J. Ye, "Tracking control for nonholonomic mobile robots: integrating the analog neural network into the backstepping technique," Neurocomputing, vol. 71, no. 16-18, pp. 3373-3378, 2008.

[2] A. M. Bloch, J. Baillieul, P. Crouch, and J. E. Marsden, Nonholonomic Mechanics and Control, Springer, Berlin, Germany, 2003.

[3] C. Samson and K. Ait-Abderrahim, "Feedback control of a nonholonomic wheeled cart in Cartesian space," in Proceedings of the IEEE International Conference on Robotics and Automation, pp. 1136-1141, Sacramento, Calif, USA, April 1991.

[4] A. De Luca and M. D. Di Benedetto, "control of nonholonomic systems via Dynamic Compensation," Kybernetica, vol. 29, no. 6, pp. 593-608, 1993.

[5] B. d'Andrea-Novel, G. Campion, and G. Bastin, "Control of nonholonomic wheeled mobile robots by state feedback linearization," International Journal of Robotics Research, vol. 14, no. 6, pp. 543-559, 1995.

[6] J. M. Yang and J. H. Kim, "Sliding mode control for trajectory tracking of nonholonomic wheeled mobile robots," IEEE Transactions on Robotics and Automation, vol. 15, no. 3, pp. 578-587, 1999.

[7] A. Bloch and S. Drakunov, "Tracking in nonholonomic dynamic systems via sliding modes," in Proceedings of the 34th IEEE Conference on Decision and Control (CDC '95), pp. 2103-2106, New Orleans, La, USA, December 1995.

[8] T. C. Lee, K. T. Song, C. H. Lee, and C. C. Teng, "Tracking control of unicycle-modeled mobile robots using a saturation feedback controller," IEEE Transactions on Control Systems Technology, vol. 9, no. 2, pp. 305-318, 2001. 
[9] T. C. Lee, K. T. Song, C. H. Lee, and C. C. Teng, "Tracking control of mobile robots using saturation feedback controller," in Proceedings of the IEEE International Conference on Robotics and Automation (ICRA '99), pp. 2639-2644, May 1999.

[10] Z. P. Jiang and H. Nijmeijer, "Tracking control of mobile robots: a case study in backstepping," Automatica, vol. 33, no. 7, pp. 1393-1399, 1997.

[11] G. Indiveri, "Kinematic time-invariant control of a 2D nonholonomic vehicle," in Proceedings of the 38th IEEE Conference on Decision and Control (CDC'99), pp. 2112-2117, December 1999.

[12] B. Ma and W. Huo, "Adaptive control of space robot system with an attitude controlled base," in Proceedings of the IEEE International Conference on Robotics and Automation, pp. 1265-1270, Nagoya, Japan, May 1995.

[13] T. Fukao, H. Nakagawa, and N. Adachi, "Adaptive tracking control of a nonholonomic mobile robot," IEEE Transactions on Robotics and Automation, vol. 16, no. 5, pp. 609-615, 2000.

[14] M. Imen, M. Mansouri, and M. A. Shoorehdeli, "Tracking control of mobile robot using ANFIS," in Proceedings of the IEEE International Conference on Mechatronics and Automation, vol. 978, pp. 4244-8115, Beijing, China, August 2011.

[15] J. Zhou, J. A. Lu, and J. Lü, "Pinning adaptive synchronization of a general complex dynamical network," Automatica, vol. 44, no. 4, pp. 996-1003, 2008.

[16] J. Lü and G. Chen, "A time-varying complex dynamical network model and its controlled synchronization criteria," IEEE Transactions on Automatic Control, vol. 50, no. 6, pp. 841-846, 2005.

[17] J. Lü, X. Yu, G. Chen, and D. Cheng, “Characterizing the synchronizability of small-world dynamical networks," IEEE Transactions on Circuits and Systems I, vol. 51, no. 4, pp. 787-796, 2004.

[18] G. Chen and X. Dong, From Chaos to Order: Perspectives, Methodologies and Applications, World Scientific, Singapore, Singapore, 1988.

[19] G. Klančar, D. Matko, and S. Blai, "A control strategy for platoons of differential drive wheeled mobile robot," Robotics and Autonomous Systems, vol. 59, no. 2, pp. 57-64, 2011.

[20] H. K. Chen, "Chaos and chaos synchronization of a symmetric gyro with linear-plus-cubic damping," Journal of Sound and Vibration, vol. 255, no. 4, pp. 719-740, 2003. 


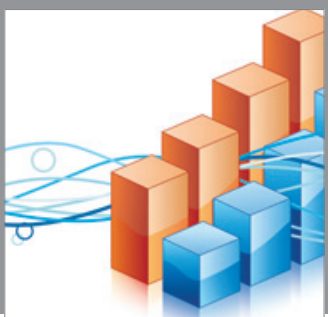

Advances in

Operations Research

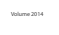

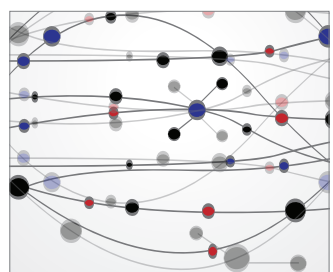

\section{The Scientific} World Journal
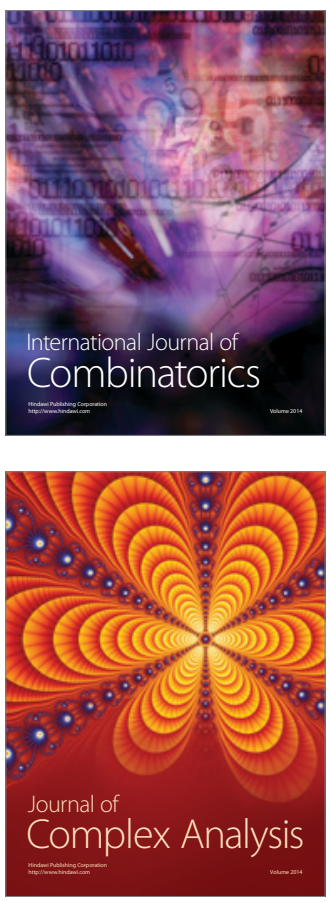

International Journal of

Mathematics and

Mathematical

Sciences
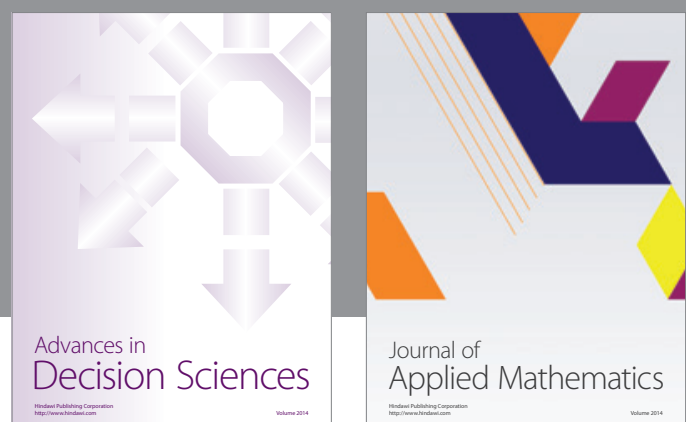

Journal of

Applied Mathematics
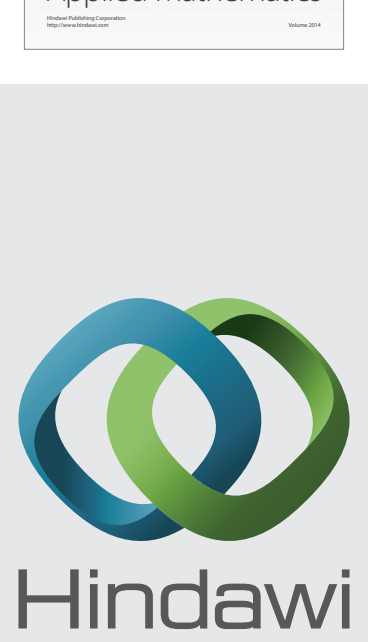

Submit your manuscripts at http://www.hindawi.com
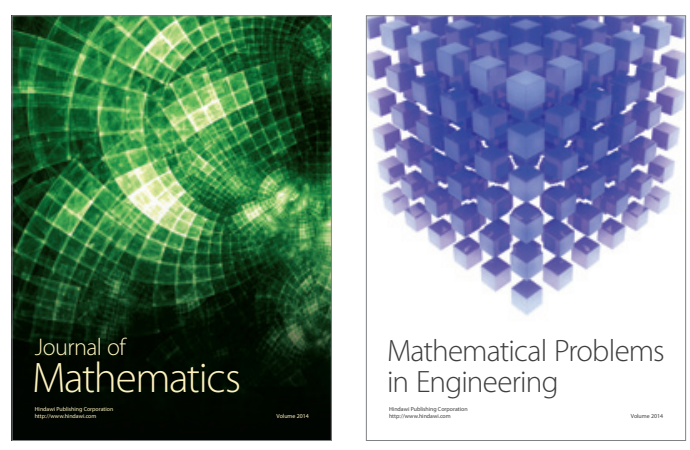

Mathematical Problems in Engineering
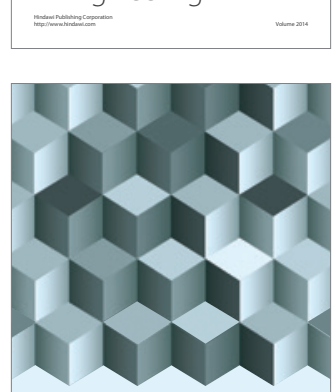

Journal of

Function Spaces
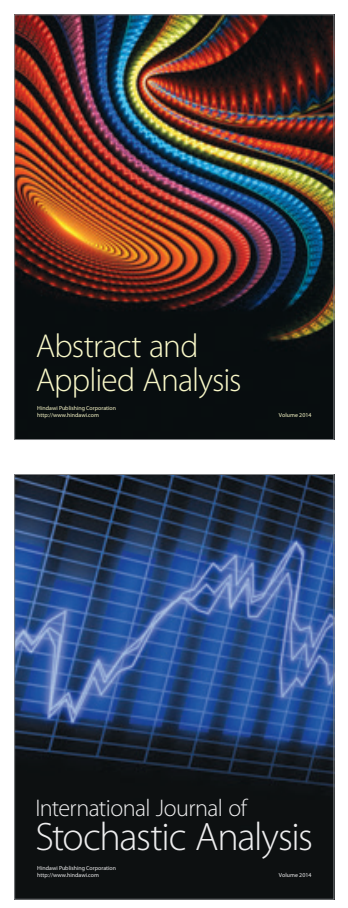

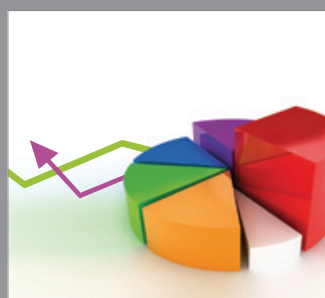

ournal of

Probability and Statistics

Promensencen
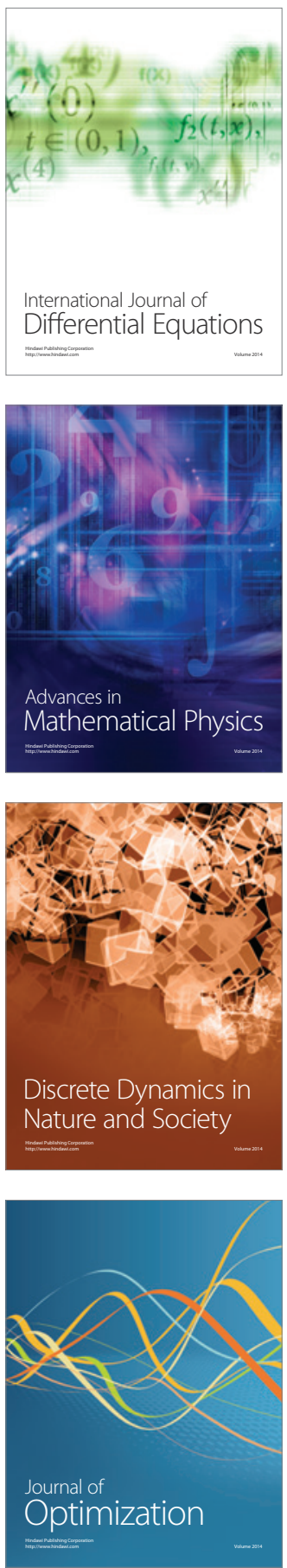